\title{
Behavioral and Epileptic Determinants of Predatory Attack Behavior in the Cat
}

\author{
R. ADAMEC
}

SUMMARY: This report presents studies which relate limbic epileptic excitability to behavional measures of defensive suppression of predatory attack in cats. Correlated with heightened defensiveness to environmental stimuli among non-killer cats is a heightened amygdaloid epileptic excitability, as well as a heightened conduction of amygdaloid epileptic activity to thalamic and hypothalamic substrates of predatory response in the amygdala to the complex visual stimuli presented by rat prey. These nearosensory responses correlate well with measures of epileptic excitability. Brain and behavior measures appear related since enhancement of excitability in the amygdala and of projection of epileptic activity by repeated electrical stimulation of predatory attacks. Furthermore, the ventral hippocampus seems capable of antagonizing the behaviorally suppressive effects of heightened amygdaloid excitability perhaps at points of convergence of amygdaloid and hippocampal output.

RÉSUMÉ: Cette étude indique qu'il existe une relation entre l'excitabilité épileptique limbique et les moyens comportementaux quiemploient les chats comme suppresssion défensive lors des attaques prédatoires. II semblerait que l'hippocampe ventral soit capable d'antagoniser les effets comportementaux suppressifs d'une excitabilité amygdalienne augmentée, possiblement à des points de convergence entre les voies provenant de l'amygdale et celles de l'hippocampe.

From the Departments of Psychology. Dalhousie University. Halifax, Nova Scotia and McGill University, Montreal, Quebec.

Reprint requests to $\mathrm{Dr}$. $R$. Adamec. Dept. of Psychology, Dalhousie University, Halifax, Nova Scotia B3H 4JI.
Goddard (1975) has suggested that long lasting changes in epileptic excitability in kindling could be used as a neural model for learning. Studies by Racine (1972b), Racine, Gartner, and Burnham (1972) and Douglas and Goddard (1975) suggest, furthermore, that changes in synaptic transmission of epileptic and non-epileptic activity accompany kindling phenomena.

Though these data are consistent with neurophysiological theories of learning which require lasting alteration of synaptic transmission within discreet neural circuits (e.g., Hebb, 1949) there have been few attempts to relate the neural changes accompanying kindling to behavioral alteration. Some changes in behavior have been reported as a result of modification of limbic epileptic excitability in the cat (Alonso de Florida \& Delgado, 1958; Delgado \& Sevillano, 1961; Grossman, 1963). In these studies, however, no clear separation of behavioral changes from seemingly pathological epileptic effects of the stimulating treatments was possible. Furthermore, McIntyre and Molino (1972) have reported only apparent dysfunctional effects of amygdala kindling on aversive conditioning. Their data suggest kindling mimics the effects of neural destruction with electrolytic lesions. These authors, however, could not determine whether the dysfunctional behavioral changes they observed were due to a lesion-like interference of amygdaloid function or to an over-activation of amygdaloid activity which might "jam" in some way the activity required for normal operation.

In order to more thoroughly test the suitability of the kindling phenomena as a model for learning, the present studies attempted to relate as closely as possible the changes accompanying limbic kindling phenomena to behavioral plasticity. Inherent in a problem like this is the need for a well defined brain-behavior model involving preferably mammalian behavior of sufficient complexity and plasticity.

Predatory behavior in the cat was chosen because it satisfied to some extent the requirements of modifiability through experience (Kuo, 1930) and of having a thoroughly investigated neural substrate (Flynn, 1967; Flynn, Vanegas, Foote \& Edwards, 1970). The work of Flynn and his co-workers suggests that several limbic system structures function either to tonically facilitate predatory attack behavior (the ventral hippocampus and dorsolateral amygdala) or to tonically inhibit it (the basal amygdala). The basal amygdala is of particular interest because it also appears to function as an excitatory modulator of defensive behavior in cats (Kaada, 1972; Stokman \& Glusman, 1970). The possibility exists that the tonic inhibitory function of the amygdala in predatory behavior is related to its excitatory role in defensive behavior. An inverse relationship between aggressive and defensive disposition is a well known concept in ethology (Hinde, 1966), and is implied for cats by Leyhausen's (1956) suggestion that non-rat-killing cats are lacking in what Schwengert (see Leyhausen, 1956) has termed "battle courage".

The relationship of defensive disposition and predatory attack was first tested by observing the behavior in response to a variety of environmental inputs of 18 cats 
which spontaneously attacked and killed hooded rats and 26 cats which would not kill rats within a $10 \mathrm{mi}$ nute exposure period. In order to obtain a number of different measures of sensitivity to threat all cats were exposed to a number of stimuli which intuitively posed environmental threats of different intensities. Responses of cats to mice, a novel environment, a human, rats, and tape recorded "threat howls" of an adult male cat were recorded on videotape in the same testing area at different times over a two month period. A dark covered corner was provided as a place to which a cat might withdraw from the threat (canopy area, Figure 1A). A variety of quantitative measures of approach, attack, withdrawal and shelter seeking were taken (e.g.,
A.

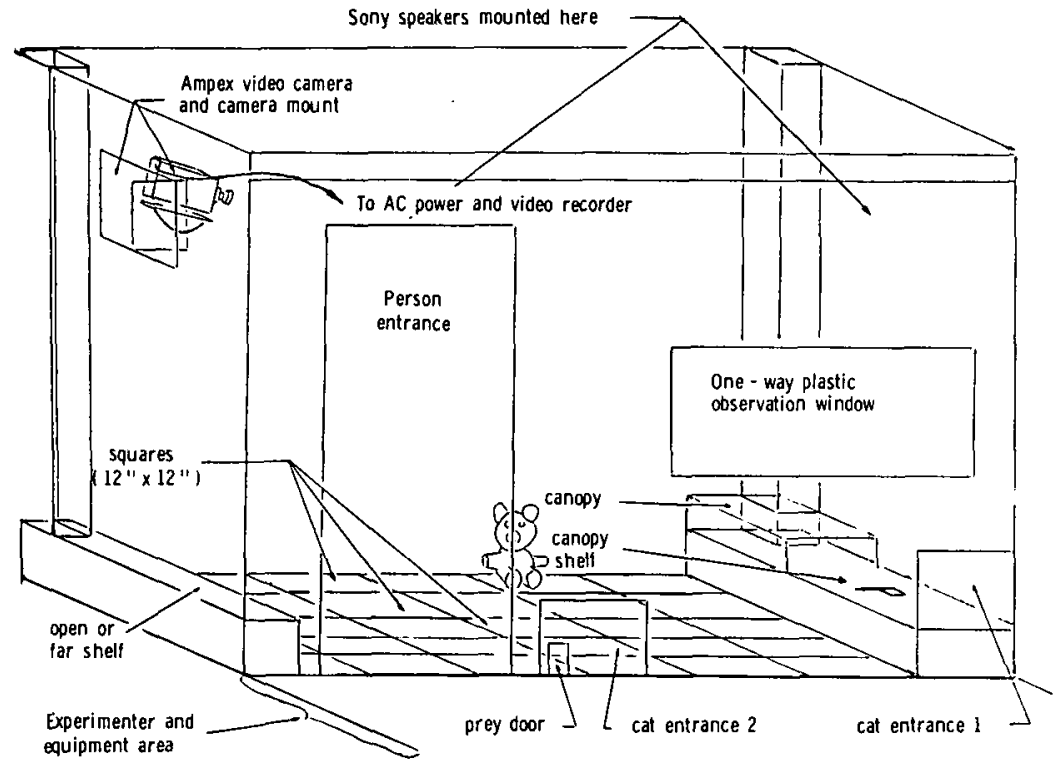

B.

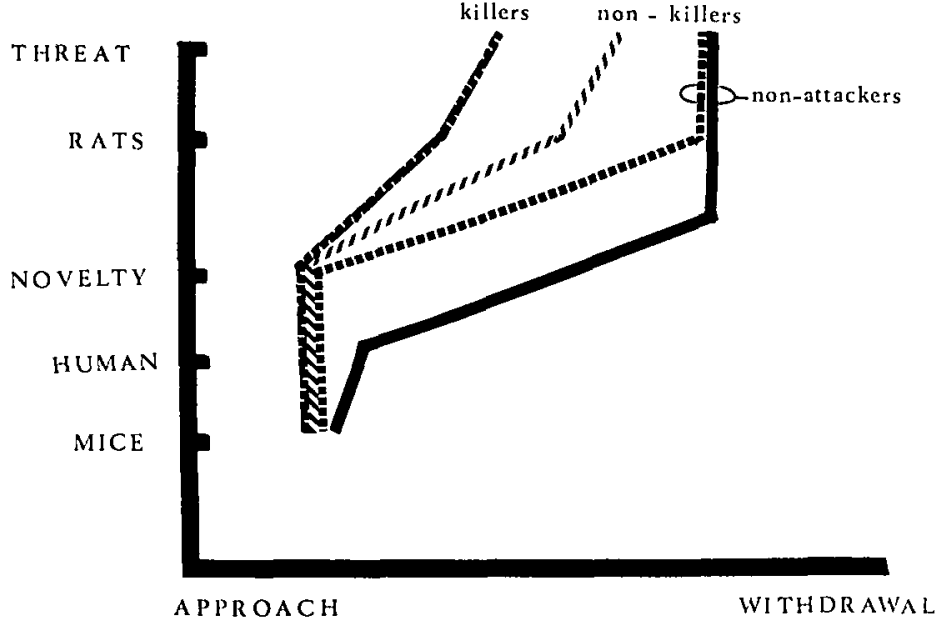

Figure I $A$-Testing area used to test responses to all environmental stimuli. The panda was only present during novelty and howl tests. No differential responses to the panda were found.

$B$-Conceptual representation of the responses of the four groups of cats to threat stimuli (see text). Stimuli are arranged on the ordinate in ascending order of supposed threat potential. A continuum of approach-withdrawal appears on the abscissa and represents quantitatively defined tendencies to approach or withdraw. Extremes of approach include active exploration of the environment, rapid tactual contact with humans, attacks on prey. Extremes of withdrawal include rapid seeking of shelter faced with any of the stimuli. latencies, durations, attack sequence scoring, such as biting-paw striking or paw-strike biting; and mode of seeking shelter, such as crouched or walking entry to the shelter area (Adamec, 1974).

Cats of differing predatory attack tendencies were found to fall along a dimension of responsiveness to environmental threat, suggesting that a personality trait of defensive responsiveness to threat varied inversely with predatory attack behavior (Figure 1B, Adamec, 1974). Four groups of cats were distinguishable. First were rat-killers $(n=18)$ which were least defensive, showing little evidence of withdrawal from any of the stimuli presented and little incidence of "defensive" paw striking attack against rats as described by Leyhausen (1956). They were affected somewhat by threat howls, though they also displayed body care behavior during the vocalizations (grooming and claw stretching) possibly displacement activities though no conflict was obvious. Furthermore, there were no after effects of the exposure to howls as measured by responses to calling and stroking.

The second group were the next least defensive. These were nonrat-killing cats which attacked rats $(n=7)$. The responses of these cats to mice, a human and a novel room were the same as those of rat killers. They differed from rat killers, however, in their response to ratprey and threat howls. Unlike rat killers, defensive paw-striking-biting attacks against rats were prevalent with biting often followed by withdrawals from the prey. Though these ambivalent attacks sometimes persisted for the full ten minute period, none of the cats injured their prey seriously (rarely any body wounds and no vertebrae damaged). Furthermore, measures of defensive paw-strike and withdrawal were highly positively correlated with biting in these animals, reflecting the alternation of attack and defensive withdrawal behavior. On the other hand, defensive measures were negatively correlated with latency to kill in rat-killers suggest- 
ing that defensive behavior interferes with a killing attack.

Rat-attacking non-rat-killers responded to threat howls by withdrawing in a crouched manner to the shelter provided and remaining there even after termination of the vocalizations. Autonomic responses like pupillodilatation, piloerection and salivation appeared in response to howls and persisted for several minutes after the howl presentation. Furthermore, after the howl presentation social responses to a human differed from those observed previously, in that the cats would not leave the shelter when called, and when physically removed they were unresponsive to stroking.

The remaining two groups of non-rat-killers did not attack rats. They approached the prey, sniffed it, backed away or withdrew abruptly from it and then went into the covered shelter area and observed the prey from a distance. Approach and sniffing showed an habituationlike decrement with repeated testing. The one non-attacking cat tested with threat howls responded in the same way as attacking nonrat-killers.

Non-rat-attackers, however, differed with respect to the "milder threats" posed by a human and novel room. One group (non-fearful non-attackers, $\mathrm{N}=13$ ) responded in much the same way to humans and novelty as killers and attacking non-killers. The remaining fearful non-attackers $(\mathrm{N}=6)$ showed greater withdrawal from humans and a novel room, spending more time in the sheltered corner than other cats and less time in active exploration of the environment or in spatial and tactual proximity to a human.

None of these groups appeared to differ in response to mice. Even the most "fearful" non-rat-killer attacked and killed mice. Because of its small size relative to the cat, the mouse, being incapable of effective self-defence, presented little threat.

It is clear from Figure $1 \mathrm{~B}$ and the above description that predatory attack tendency varies with sensitivity to threat as measured by prevalence of withdrawal and defensive attack in response to environmental stimuli. It remains to be demonstrated whether the excitability of the amygdala can be related in a meaningful fashion to this behavioral trait of sensitivity to threat.

To do this, the relationship between behavioral measures of attack and withdrawal and threshold for electrically elicited after-discharge (AD) in the amygdala as well as propagation of $A D$ to other limbic system areas were examined. The AD threshold (ADT) was chosen because Racine (1972a) has shown it to be a stable measure of epileptic excitability which is also experimentally modifiable.

Twelve rat-killers and fourteen non-rat-killers were randomly selected from the above behavioral experiment. While under combined acepromazine mateat (atravet) and Nembutal $(30 \mathrm{mg} / \mathrm{kg}$ ) anaesthesia, all cats were implanted with two to four twisted bipolar platinum-10\% iridium electrodes $(.007$ inches in diameter with a .003 inch coating of teflon and a $.5 \mathrm{~mm}$ tip separation) using sterile stereotaxic procedures (Kopf stereotaxic instrumént). Electrodes were aimed at the basomedial amygdala, anterior ventral hippocampus, medial thalamus and medial hypothalamus (Figure 2). These thalamic and hypothalamic areas were known to inhibit predatory attack in these cats when stimulated directly with $62 \mathrm{~Hz}$ biphasic 1 msec. rectangular pulses just prior to sacrifice (Adamec, 1974).

Following four weeks of postsurgical recovery, cats were re-
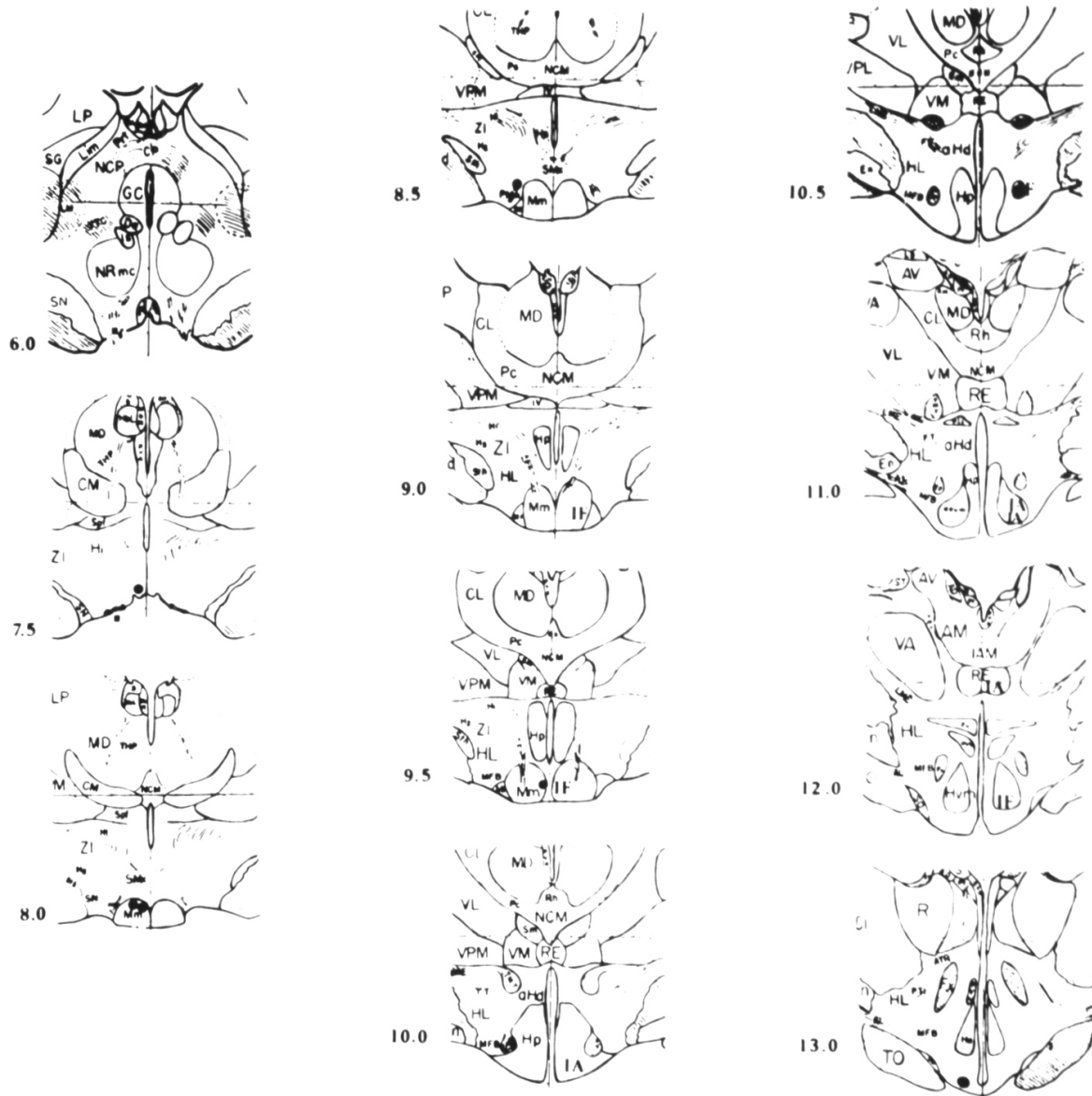

Figure 2-Electrode placements in thalamic and hypothalamic areas which suppress preying. Points are projected onto sections of the Jasper and Ajmone Marsan cat brain atlas. Numbers by each section are the frontal plane of that section. Non-killer placements appear as black dots on the left, killer placements on the right. The 5 "IA" placements on the right refer to electrodes on inhibited rat-killers. "IF" refer to placements in facilitated killers stimulated in the ventral hippocampus. Abbreviations are according to Jasper and Ajmone Marsan. 
tested for their responses to prey and humans. No surgical effects on the behavior were found.

After discharge threshold (ADT) was then determined to within \pm $50 \mu \mathrm{A}$ or $100 \mu \mathrm{A}$. It was defined as the minimal peak-to-peak intensity of a $3 \mathrm{sec}$. train of $60 \mathrm{hz}$ constant current biphasic rectangular pulses of $1 \mathrm{msec}$. pulse width necessary to elicit an electrical epileptic discharge (AD) outlasting the stimulus.

Stimulation was begun at an initial current level of $400 \mu$ A peakto-peak. If an $\mathrm{AD}$ was elicited at this first intensity, the current was decreased in $200 \mu \mathrm{A}$ and $100 \mu \mathrm{A}$ and then $50 \mu \mathrm{A}$ steps. Otherwise, the intensity was raised in $400 \mu \mathrm{A}$ steps until an AD was elicited. The current was then reduced in 100 $\mu \mathrm{A}$ steps until an $\mathrm{AD}$ was no longer seen. The minimal stimulus intensity needed to trigger an $A D$

PRE

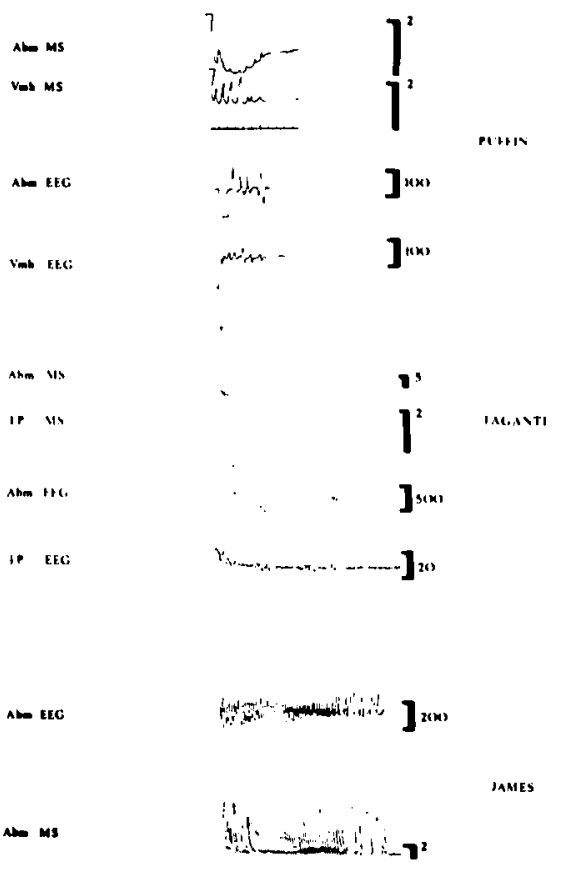

Figure 3-Examples of ADs recorded prior to suppression of killing at AD1 (PRE) and after suppression of preying (POST) with stimulation of the amygdala in two ratkillers (Piffin and Jaganti). ADI of one non-rat-killer appears for comparison. The top of the vertical lines opposite records of mean square (MS) activity indicate levels 2-5 times the pre-stimulation baseline MS level. Lines opposite EEG traces indicate pen displacement necessary to reflect a voltage change (in $\mu v$ ) as large as the number indicated. Time markers indicate time in seconds. Activity was recorded simultaneously in the amygdala $(\mathrm{Abm})$ and interpeduncular nucleus (IP) or ventromedial hypothalamus (Vmh).
POST

was considered the threshold. A maximum of six stimulations, separated by one hour, were given each day. A 24 hour interval was allowed between threshold determinations in different structures. Only amygoid and hippocampal thresholds were not elicited.

Stimulating pulses were generated with a Grass S8 stimulator, isolation and constant current units. EEG activity and integrated multiareas with a Grass model 7 polygraph. MS activity was differentially amplified (Teketronics 2A61 amplifiers) and integrated with Ballantyne mean square meters (Figure 3 ). MS data were reduced to the average amplitude of MS spikes (above

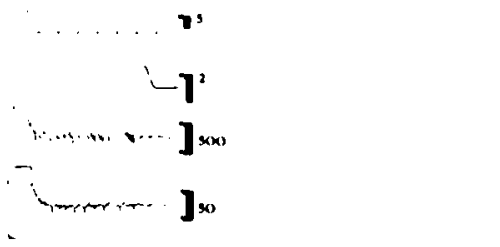

noise level of the polygraph) during $\mathrm{AD}$ and this average was expressed as a percentage of the average MS level recorded (over a 2 minute period) prior to any brain stimulation. A measure of EEG frequency was also taken as the average number of changes of direction of voltage per second during an AD.

After discharge threshold (ADT) in the basolateral amygdala (magnocellular and parvocellular divisions) was found to vary with the behaviorally defined continuum of sensitivity to threat. Fearful cats had the lowest thresholds (mean $130 \mu \mathrm{A} . \mathrm{N}=3$ ). These thresholds did not overlap with the higher thresholds of unfearful non-ratattacking cats (mean $360 \mu \mathrm{A}, \mathrm{N}=$ 5). Furthermore, non-killing ratattacking cats had higher thresholds than either of the groups of nonrat-attackers (mean $430 \mu \mathrm{A}, \mathrm{N}=$ 4), again without overlap. Finally, rat killers had the highest thresholds (ranging from $800 \mu \mathrm{A}$ to $1800 \mu \mathrm{A}$, $\mathrm{N}=11)$ which also did not overlap with any of the non-killer thresholds. These threshold differences are not attributable to differences in location of electrodes of these groups (Adamec, 1975). Nor are they a function of general brain excitability differences, since no differences in thresholds in the white matter lateral to the amygdala were found (killers range $600 \mu \mathrm{A}$ to 1800 $\mu A, N=5$; non killers range 600 $\mu \mathrm{A}$ to $800 \mu \mathrm{A}, \mathrm{N}=4$, not significantly different) or in ventral hippocampal thresholds (killers range $200 \mu \mathrm{A}$ to $500 \mu \mathrm{A}, \mathrm{N}=5$; nonkillers range $200 \mu \mathrm{A}$ to $700 \mu \mathrm{A}, \mathrm{N}$ $=12$, not significantly different).

Of equal interest was the finding that MS measures recorded within the amygdala and propagated from the amygdala to thalamic and hypothalamic areas during the first AD were even better predictors (correlates) than the ADT of measures of defensive behavior in all test situations. For example: greater activity propagated from the amygdala to the medial thalamic and hypothalamic areas was associated with more prolonged withdrawal from the environmental threats of novelty and threat howls and grea- 
ter defensive attack behavior toward rats. Similarly EEG frequency of amygdaloid $A D$ of non-killers was higher and correlated well with measures of withdrawal (Adamec, 1974); range of correlations $.60-1.00$.

If these measures of neural activity during an $A D$ have any functional significance, then changing them in rat killers ought to inhibit predatory behavior. This in fact was observed. One to 19 days of once daily stimulation of the amygdala of rat killers $(3 \mathrm{sec}$. bursts of the threshold determining stimulus kept at $400 \mu \mathrm{A}$ below the ADT as it decreased) lowered their ADTs to within the range of non-killers, and inhibited their tendencies to kill rats. In no case were they stimulated in the presence of a rat. Changes in predatory behavior appeared abruptly. The attack pattern then remained stable over subsequent tests. The inhibition lasted as long as the cats were kept alive: 6 days in 2 cats, 20 days in 1 cat and $1-3$ months in 4 cats. Accompanying the inhibition of killing were behavioral changes indicating an increase in defensive responses to rats and nonspecific threat only. All cats showed increases in paw striking attacks, decreases in biting and increases in withdrawals from rat prey as well as elevated and more prolonged autonomic responses to threat howls (with respect to their presurgical behavior and unstimulated controls). The fact that changes in response were limited to these two stimulus situations (response to novelty could not be tested) is consistent with the observations that nearly all measures of the predatory behavior of stimulated cats were the same as those of attacking non-rat-killers - the next to least defensive cats on the defensive dimension (Adamec, 1974).

In addition to behavioral and ADT similarities, all other AD parameters in killers came to resemble those of non-killers with suppression of preying. Of greatest interest was the fact that measures of propagation of neural activity during an $A D$ changed in such a way as to suggest that active altera- tion of transsynaptic propagation of neural activity coincided with the behavioral changes. Changes in average MS values were assessed in all areas by examining 5 successive amygdaloid ADs recorded as ADTs were decreasing. The first three ADs were: AD1 - first recorded $\mathrm{AD}$; $\mathrm{AD} 2$ - an $\mathrm{AD}$ observed usually after one week of threshold lowering stimulation; AD3 - the $A D$ recorded on the day before behavioral change (usually one week after AD2). AD4 was recorded on the first day of behavior change. AD5 was recorded six days later after daily behavioral tests of response to prey (rats and mice) and humans. There was no brain stimulation between ADs 4 and 5 .

Activity during $\mathrm{AD}$ in all areas was measured as the average level as described above. In addition, MS values in thalamic and hypothalamic areas were expressed as a ratio of the MS value in the amygdala (Eo/Ei).
As ADT decreased integrated neural activity during an $A D$ in the amygdala rose gradually, peaking at AD3, before any behavioral change and before any significant change in propagation of neural activity (Figure 4). MS levels in thalamic and hypothalamic areas rose abruptly at AD4 - on the same day as the behavioral changes - persisting throughout the suppression of predatory behavior (Figure 4). Furthermore, when propagated activity increased, there appeared, for the first time, a correlation between frequency of epileptic spiking of both the integrated MS and EEG activity of the amygdala and thalamic and hypothalamic areas (Spearmann's $\rho .90$ to $1.00, \mathrm{~N}=5$ ). These data suggest establishment of more direct communication between the amygdala and these medial structures. Moreover, coincident with these propagated activity increases was an increase in EEG frequency in the amygdala at AD4.

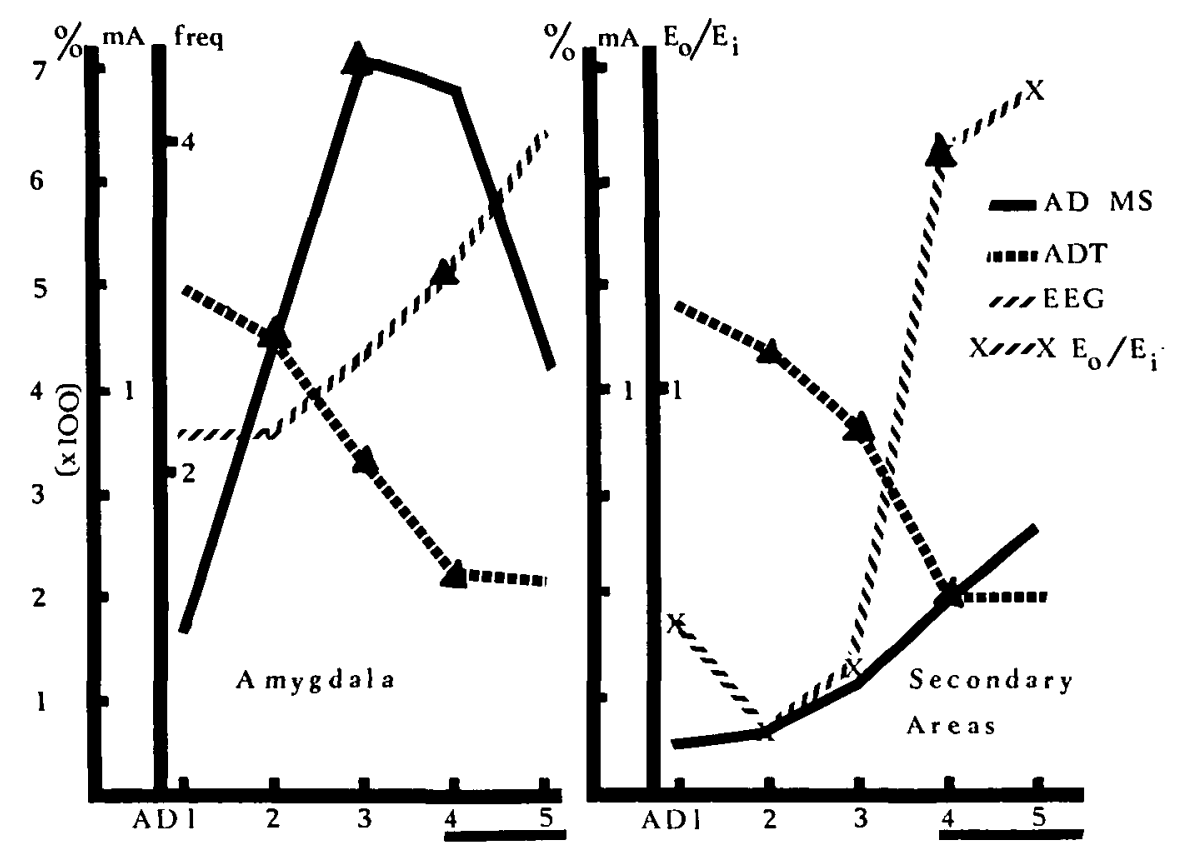

Figure 4-Graphs of amygdala, thalamic and hypothalamic area AD parameters which change with repeated stimulation of the amygdala. Values plotted across ADs 1-5 are averages across cats of: mean square activity during $A D$.(AD MS) as a percentage of first recorded prestimulation MS baseline; ADT in $\mathrm{mA}$; EEG frequency: and ratio of thalamic and hypothalamic MS activity to simultaneously recorded amygdaloid activity (Eo/Ei). Changes were assessed by Friedman analysis of variance by ranks $(\mathrm{p}<.05)$. A triangle indicates that point differs from the preceding $\operatorname{AD}(\mathrm{p}<$ .04 , sign test). The line under ADs 4 and 5 indicate ADs taken after behavior was observed to change. 
Since this measure reflects both EEG frequency and complexity (Figure 4), the increase could indicate an increased feedback from thalamic and hypothalamic areas over physiologically and anatomically defined pathways (e.g., Happel \& Bach, 1970). There were no EEG changes in thalamic and hypothalamic areas, however. These data suggest a strengthening of pre-existing connections rather than addition of new ones, or elimination of old ones.

Transsynaptic connectivity may thus be the most behaviorally relevant neural dimension. In the first place, evidence of enhanced transmission was found during the first amygdaloid AD of non-rat-killers. Secondly, Racine et al., (1973) have suggestive evoked potential evidence that transsynaptic changes following repeated evocation of epileptic activity appear in non-epileptic driving of the same amygdaloid circuits in rats.

The effects of hippocampal stimulation on brain and behavior lend further credence to the functional

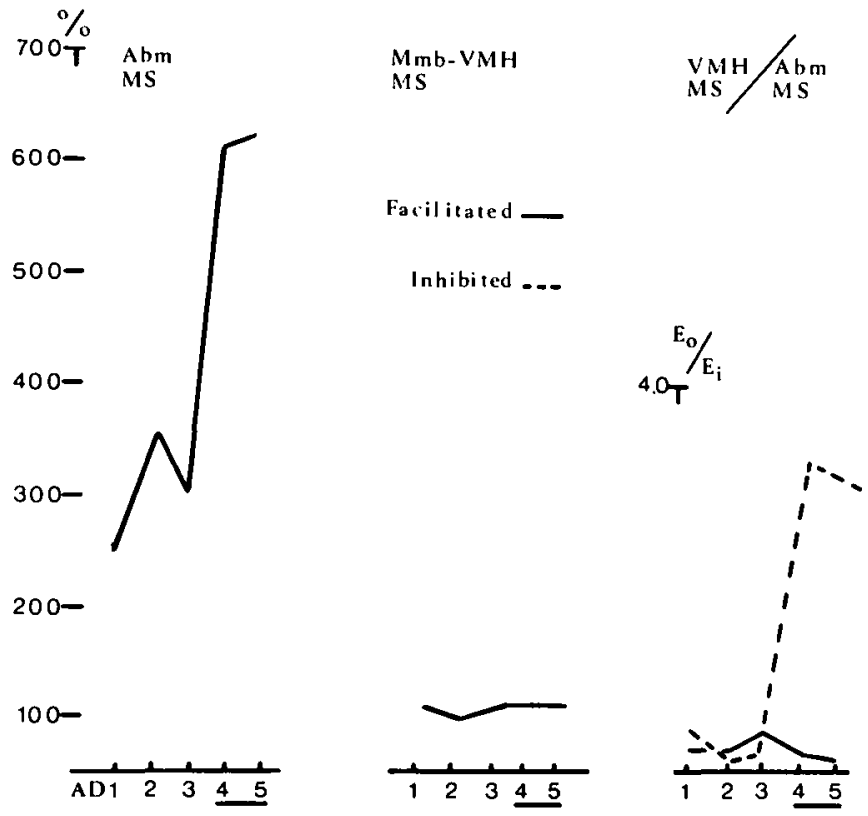

Figure 5-Graph of averages of MS activity changes across ADs $1-5$ in facilitated killers $(n=3)$. Amygdaloid $(\mathrm{Abm})$ changes are significant, (Friedman Analysis of variance, $\mathrm{p}<$ $.05)$ but mammillary body $(\mathrm{Mmb}, \mathrm{n}=2)$ and ventromedial hypothalamic (VMH, $\mathrm{N}=1$ ) changes are not. The last graph compares $\mathrm{VMH}$ response in one facilitated cat with $\mathrm{VMH}$ response in two inhibited killers. significance of transsynaptic alteration. Four rat-killing cats were stimulated repeatedly in the ventral hippocampus, the area tonically facilitating attack according to Siegal and Flynn (1968). All 4 showed an increased speed of killing prey, and decreasing trends in defensive responses to prey. Three of these 4 were also alternately stimulated in the contralateral amygdala as many times as were inhibited killers and had as many amygdaloid ADs. Yet they showed a permanent facilitation rather than inhibition of predatory behavior. Examination of the nature of their amygdaloid ADs revealed no change in MS activity propagated to hypothalamic areas (mammillary bodies and ventromedial hypothalamus) during amygdaloid AD though amygdaloid neural activity during AD grew (Figure 5). Furthermore, there was no growth in EEG frequency of amygdaloid $A D$, sug- gesting no increase in feedback. It should be mentioned that 2-7 hippocampal ADs preceded amygdaloid stimulation.

These data suggest that ventral hippocampal activation blocked changes in transsynaptic connectivity associated with suppression of attack. The blocking action most probably is at sites of convergence of amygdaloid and hippocampal output, such as the medial hypothalamus (Van Atta \& Sutin, 1972) since no changes in hippocampal AD parameters were observed.

In order to test if behavioral changes resulting from brain stimulation were reversible, inhibited killers $(N=4)$ were cyclically deprived of food 20 days after suppression of preying was observed. An additional four unstimulated cats $(2 \mathrm{kil}-$ lers and 2 non-killers) were also deprived. Previous experiments in rats (Paul, Miley \& Baenninger, 1971;

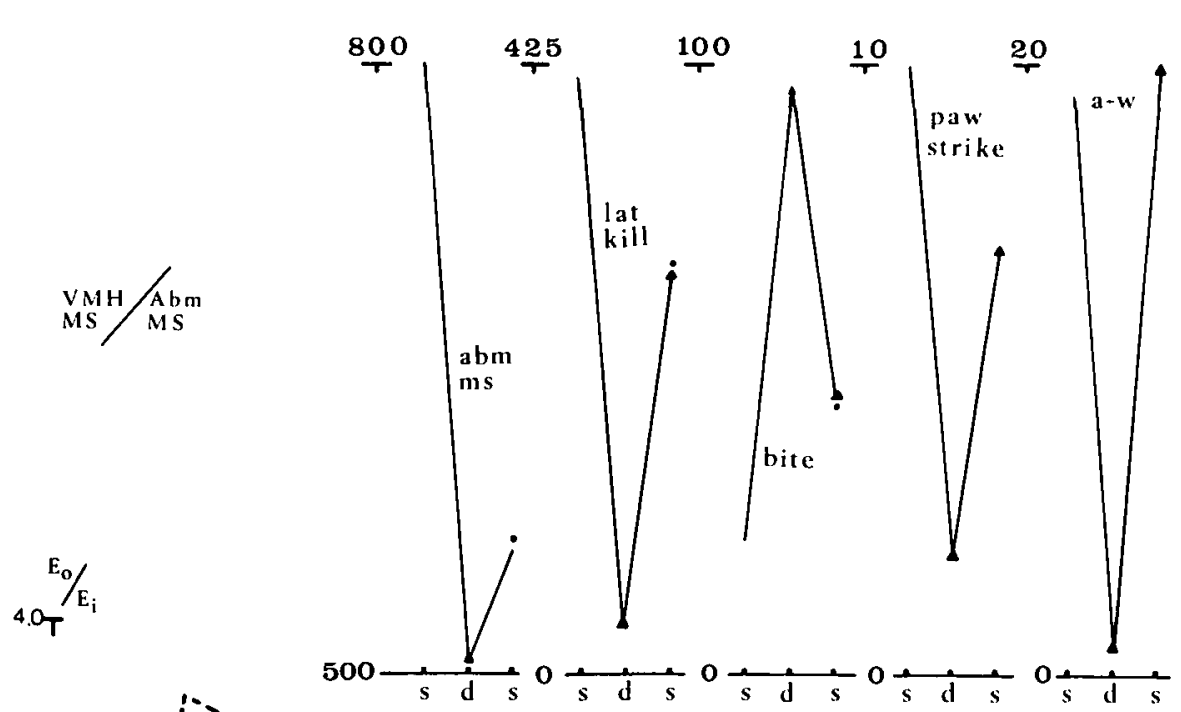

Figure 6-Graphs contrasting neural response in the amygdala during $\mathrm{AD}$ ( $\mathrm{Abm} \mathrm{MS}$ ) with changes in attack response to rats during satiation, food deprivation-satiation testing (sds on the abscissa). Neural activity is average mean square (MS) level as a percentage of the first recorded pre-stimulation baseline. Behavioral measures are: kill latency in seconds (lat kill); time biting prey as a percentage of time near the prey (bite); time paw striking prey as a percentage of time near (paw strike); and frequency of approach-withdrawals. Data are means across cats. Variation across testing periods are all significant (analysis of variance $p<.01$, approachwithdrawals and paw strike analysis used log transformations). A triangle indicates the plotted point differs from the previous point, and a dot indicates a difference from the first plotted point (studentized range statistic, all significant (s-d comparisons), $\mathrm{p}<.01$, all significant $\mathrm{s}-\mathrm{s}$ comparisons $\mathrm{p}<.05$, Winer, 1962). 
Adamec \& Himes, 1975) and cats (Adamec, unpublished observations) suggested that food deprivation should induce predatory attack in non-predators.

The sequence of testing was comprised of four periods. In the first period response to rats was tested while cats were on an ad lib feeding regime. One hour later an amygdaloid $A D$ was elicited at threshold intensity and activity in the amygdala and hypothalamic areas monitored as previously described (Hvm, Mm,Hp,Ip-Fig. 2).

Some cats had ventral hippocampal placements. One cat (a nonkiller) was stimulated in the ventral hippocampus alone and three (a non-killer and two killers) with both hippocampal and amygdaloid electrodes were stimulated in the hippocampus one hour after amygdala stimulation. Cats were not tested again for a time control period which equalled the entire time period encompassed by the next three tests ( 4 to 28 days). Cats were again tested in the manner described above. They were then placed on a 48 hour food deprivation schedule for 2 to 14 days. Prey response and neural response during $A D$ were again measured. Cats were then allowed ad lib feeding for the same number of days as the days of deprivation. Prey response and AD again were measured.

Food deprivation reinstated attack in all inhibited killers and natural non-killers, and facilitated attack in the rat-killers. One of the inhibited killers and one of the non-killers continued to kill during subsequent resatiation. Quantitative analysis revealed a decrease in kill latency and an increase in biting in all cats during deprivation (Figure 6). There was a concomittant decrease in measures of attack ambivalence (approach followed by rapid withdrawals from prey, Figure 6) and of defensive paw striking attack (after Leyhausen, 1956). Attack deteriorated somewhat with resatiation in all cats, with an elevation of kill latency and of defensive measures and a decrease in biting. Nevertheless, kill latency remained lower than the initial satiated control period in those cats which persisted in killing. All cats also bit the prey significantly longer upon resatiation.

Integrated neural response in the amygdala during AD parallelled these behavior changes though ADT did not change (Figure 6). ADT and neural activity in the ventral hippocampus, and activity propagated
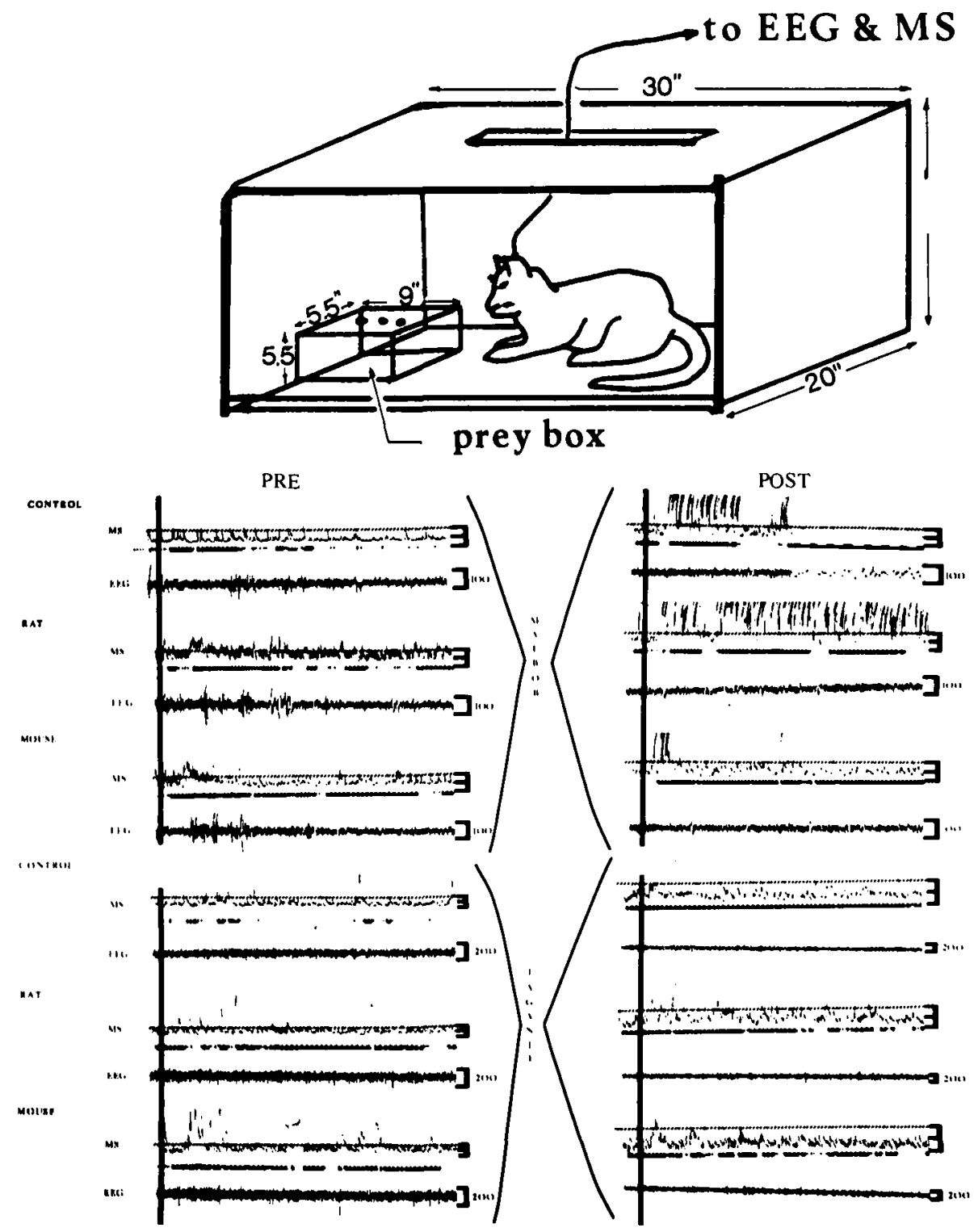

Figure 7-Top of the figure is the recording box in which prey were presented for cats to view. On the bottom are examples of mean square (MS) records taken from the amygdalas of two rat killers prior to brain stimulation (PRE) and after suppression of preying with amygdaloid stimulation (POST). Records are samples from successive periods of slide control, rat and mouse presentation (see text). The reverse " $E$ " markings beside MS records mark MS levels equal to or twice the baseline activity recorded for 5 minutes prior to the slide control. Calibration for EEG is indicated by EEG records in $\mu \mathrm{V}$. Time markers appear between $\mathrm{MS}$ and EEG records. Behavioral event markers are superimposed on the time markers and are: visually oriented to prey box; 17 paw strike prey box; movements. The vertical line across all records indicates when the plastic slide was inserted (see text). 
by reducing amygdaloid excitability alone. Accompanying this reduction is a reduction in defensive responses to prey which normally interfere with attack. Biting attack persists following deprivation and amygdaloid excitability remains lowered. The re-appearance of defensive responses following deprivation may be a function of persistent biting attack, since attack elicits prey selfdefence which in turn elicits predator defensive response (Adamec, 1974; DeFeudis, 1975). Since predator defensive response following deprivation is no greater than before deprivation, in spite of heightened prey reaction to more intense attack, there may have been a net decrease in predator defensiveness.

Taken together the data support the hypothesis that these measures of epileptic excitability of limbic circuits are measuring a mechanism of bias of responsiveness of restricted limbic circuits to input which in turn biases the cats' response to those inputs. In view of the evidence for complex sensory response of amygdaloid cells (O'Keefe \& Bouma, 1969) an attempt was made to compare sensory response of these various brain areas in both rat-killers and non-rat-killers.

Initial recordings of activity were taken prior to any brain stimulation. Cats were thoroughly familiarized with the recording box, having spent at least 8 hours in it with electrode leads attached (Figure 7). During recording of brain activity, cats were presented with prey they could see and smell, but not physically contact, by sliding prey into the transparent prey box with a plastic slide. This exposure to prey lasted for 5 minutes, followed by an additional five minutes of recording after the slide containing prey was removed.

Prior to prey presentation, the empty plastic slide was placed in the prey box for $90 \mathrm{sec}$. to obtain a measure of neural response to all potentially arousing effects of introducing the slide alone. All measures of response to prey were taken as a ratio of the arousal response produced by the slide alone.

Amygdaloid and hippocampal activity was differentially recorded be- tween the tips of the bipolar electrodes, integrated with Ballantyne mean square meters and the dc output displayed on chart paper with a Grass model 7 polygraph. Hypothalamic activity was recorded on the next day (Ip, Mm, medial hypothalamus, PMn, Hvm- Figure 2). Event markers were used to simultaneously indicate the behavior of the cat on the polygraph record (Figure 7).

Analysis of the records was done blind by a rater with no knowledge of the experiment. Average level of integrated activity for each $10 \mathrm{sec}$. interval was determined as the midpoint of a visually fitted straight line through the activity. These levels were sorted into behavioral categories of paw striking, visual orientation to the prey box and looking elsewhere.

An example of the observed MS activity appears in Figure 7. It is clear that there was a neural response in the amygdala to a rat stimulus when the cat first visually oriented (see time marker legend in the figure). This impression was borne out statistically as well (comparison of MS levels during first 10 sec. of orientation to slide with first $10 \mathrm{sec}$. of orientation to rat, correlated $\mathrm{t}=2.994, \mathrm{df}=6, \mathrm{p}<.05$ ). Furthermore, a similar visual response to prey was seen in the ventral hippocampus (correlated $\mathrm{t}=$ $2.040, \mathrm{df}=10, \mathrm{p}<.05)$. No clear sensory responses were recorded in hypothalamic areas of killers and non-killers, however (Hvm, Hp, Mm - Figure 2). The response in the amygdala also appeared to be heightened following suppression of preying in the two stimulated killers for whom these data were available (Figure 7). Note that there is no epileptic activity evident in the records of inhibited killers. There may also have been a differential response to mouse prey. Since mice were always presented last, it could not be ascertained whether the diminished response to mice was a function of habituation or a property of the prey stimulus.

Comparisons of killers and nonkillers revealed the integrated neural response of the amygdala of non- rat-killers was higher than that of killers when the cats visually oriented to a live rat $(\mathrm{t}=3.461$, $\mathrm{df}=6$, $\mathrm{p}<.01,5$ killers, 3 non-killers). This response appeared to persist for as long as several minutes after the rat was removed (comparisons of average MS levels after prey were removed, $\mathrm{t}=4.915$, $\mathrm{df}=6, \mathrm{p}<.01$ ). The greater activity in the amygdala of non-killers is probably dependent upon sensory input in as much as killers and non-killers do not differ with regard to activity level during paw striking. Similar measures of activity within the ventral hippocampus revealed no differences (6 killers and 6 non-killers). Behaviorally related neural activity differences to sensory input, then, appear restricted to the amygdala and are not reflective of differences in general excitability.

Of further interest is the correlation of amygdaloid response during prey orientation with ADT (Kendall's $\tau=-.841$ ) and with average MS activity generated during the first $A D$ (Pearsons' $r=.717$ ). The correlations suggest cats with lower thresholds and greater MS during $\mathrm{AD}$ show greater amygdaloid response both to immediately sensory input and in persistence of response after prey removal. Since ADT was determined after recording these data, these correlations suggest some connection between epileptic excitability and normal excitability. No such correlations were found with respect to the ventral hippocampus.

With regard to facilitated killers, there was no change in hippocampal sensory response to rats following behavioral changes (4 cats and 5 placements). There was a trend, however, toward a depression of amygdaloid response (3 cats, 3 placements) in the first $90 \mathrm{sec}$. of viewing the rat (comparing each 10 sec. interval from pre and post stimulation records, sign test, $p<.02)$. These data suggest that one site of hippocampal action in blocking amygdaloid suppression may be within the amygdala itself or via inhibitory feedback as a consequence of hippocampal output. Of relevance is the fact that EEG frequency of $A D$ triggered in the amygdala of facili- 
tated killers was nearly identical to the EEG frequencies of their hippocampal ADs triggered an hour apart (averages across ADs 1-5 are amygdala, 10; hippocampus, 10.2). These amygdaloid and hippocampal frequencies of facilitated killers were higher than even the highest amygdaloid EEG frequencies of inhibited killers recorded at AD5 $(\mathrm{t}=2.700$, $\mathrm{df}=8, \mathrm{p}<.05$ ). Apparently the hippocampal rhythm dominated seizure activity even when AD was elicited from the amygdala.

Since the above data are based on small numbers of animals, conclusions drawn must remain tentative. Nevertheless, they are consistent with the hypothesis that amygdaloid response to complex sensory input relevant to preying biases response to prey. Non-rat-killers which show greater amygdaloid response to complex input, most probably do not kill because of the greater activity generated in the amygdala and perhaps transmitted to attack suppressive areas when prey is presented. The nature of this transmission may be more of a biasing than a driving input to these areas. The apparent prolonged neural response in the amygdala after the prey is removed is reminiscent of the prolonged withdrawal responses of non-killers to other environmental stimuli. Since integrated neural responses to stimuli other than prey were not recorded, the relationship of amygdaloid response to threatening stimuli in general is not known. A relationship is implied, however, since there were good correlations between measures of epileptic activity during $\mathrm{ADI}$ and withdrawal tendencies in all threat situations.

Finally, the lack of difference in hippocampal sensory response between killers and non-killers does not preclude differences in impact of hippocampal output in distal areas. Such a mechanism is suggested by the stimulation data and to some extent by the neurosensory response data. Further work on hippocampal amygdala interaction at points of convergence is obviously needed.

$$
\text { CONCLUSIONS }
$$

The data suggest that non-ratkillers are more sensitive to a vari- ety of environmental threats. Greater defensiveness appears to interfere with attack on rat prey. Correlated with heightened defensiveness to all stimuli is a heightened conduction of such activity to thalamic and hypothalamic substrates of predatory suppression. These measures of excitability appear to be causally related to the behavior since modification of local amygdaloid excitability and of transsynaptic conduction with repeated electrical stimulation modifies the behavior. The ventral hippocampus seems capable of antagonizing the behaviorally suppressive effects of heightened amygdaloid excitability, perhaps at points of convergence of amygdaloid and hippocampal output.

Though excitability is assessed by generation of abnormally synchronous (epileptic) activity, there are reasons to believe these measures reflect normal function. First, naturally occurring differences between rat-killers and non-killers in measures of epileptic excitability exist and correlate with the behavior. Second, all AD parameters which were found to differ between killers and non-killers, i.e., ADT, EEG frequency, MS activity in the amygdala and propagation of MS activity to other areas, were observed to change with behavioral changes. These parameters of killers inhibited with brain stimulation all came to resemble the parameters of non-killers. Third, temporary reversal of predatory suppression by a normal alteration of physiological state (hunger) is reflected in a decreased epileptic excitability of the amygdala. Fourth, amygdaloid neural response to complex sensory input is greater in natural non-killers and correlates well with subsequently assessed epileptic excitability.

These data support the hypothesis that epileptic excitability and its modification by repeated stimulation is a behaviorally relevant model of neuro-behavioral plasticity.

Since predatory behavior is experientially modifiable (Kuo, 1930), kindling and ADT modification may be useful as methods of investigating the impact of experience on the brain.

\section{DISCUSSION}

Dr. McIntyre: What would you think would happen during kindling of the VMH? Do you think that it would have any influence on the amygdala? Dr. Adamec: I would think that it might. It might influence the amygdala because you generate a good deal of feedback. conceivably over the stria terminalis which does have excitatory inputs to the amygdala. In fact, you might kindle the amygdala via the VMH. Dr. McIntyre: If feedback were eliminated by sectioning, would you think that stimulation of the $\mathrm{VMH}$ alone could have an influence? Dr. Adamec: It might. I have some data on one animal in whom I was stimulating the mammillary bodies. Stimulation at this point produced stimulus-bound suppression of predatory attack. Repeated stimulation of the mammillary bodies in the presence of prey in this cat leads to a permanent suppression of its attack upon mice. That may be evidence that stimulation of some of these secondary areas may produce the same effect as stimulation of the amygdala. Dr. McIntyre: Graham reports there are data on this that I don't know of. Could you tell me what you think the influence is of something like norepinephrine on the non-killers. Dr. Adamec: There is some equivocal evidence of Leaf's that norepinephrine may suppress kindling in that depleting agents systemically injected may facilitate killing in rats. The facilitation is unreliable, however. Imipramine, which may interfere with re-uptake of norepinephrine. blocks killing when injected into the rat amygdala. These effects may be related to an antagonism between adrenergic and cholinergic activity in the amygdala. Ben-Ari has shown the highest adrenergic and dopaminergic concentrations to be in the central and basolateral nuclei of the amygdala. These areas seem to facilitate killing in rats, since Karli has shown that lesioning them suppresses attack. Furthermore, Ebel and his coworkers find higher choline acetyl-transferase activity in the amygdala of killer rats. These last data are consistent with earlier work of Smith, King and Hoebel showing a possible cholinergic basis for hypothalamic mediation of attack behavior. All of these data may be inappropriate to the cat. Cholinergic stimulation of the hypothalamus or of the amygdala produce defensive behavioral episodes. a behavior which I am suggesting is incompatible with predatory attack. Furthermore. Karli suggests the centromedial. basolateral amygdala facilitates killing in rats, whereas Egger and Flynn find the opposite in cats. Species differences may then be a real possibility and a problem. Dr. Phillips: The suggestion has been made that abnormal seizure discharge in the amygdala may underlie some displays of excessive aggression by humans. Do you see your work on aggression in cats as having any bearing on this issue? Dr. Adamec: There are two points I would like to make: First of all. I did not see any clear evidence of epileptic discharging to sensory input in the inhibited cats. Secondly, predatory attack on the cat seems to be a very unemotional behavior and may not be an appropriate model for excessive and emotional human reactions. With respect to studies of elicited rage or aggressiveness there 
is more than one reason why an organism might show an "emotional" attack behavior Adopting Moyer's point of view, one would have to admit to the possibility of numerous types of aggressive reactions, differing with respect to environmental and motivational control and with respect to neural substrate. For example, an aggressive reaction might paradoxically be a manifestation of extreme fear. You can make an organism feel so fearful that it will attack vehemently in defense of itself. This has been described in ethology as the critical reaction especially if an animal is cornered. Descriptions of rage responses associated with heightened amygdala activity 1 don't think, separate between the possibility that it might be aggression in the sense of maybe the cold calculated aggression of a killer cat or human and the possibility that there may be an intense terror episode or some other source of the aggressive behavior. $D r$. Fernandez-Guardiola: 1 do not consider the behavior of your "killer cats" as a violent behavior. We all agree I believe, that this is a normal predatory behavior for the feline species. Your results are not so surprising if we focus the behavior in terms of attention and vigilance on one side and distraction and somnolence on the other. Arousal as a consequence of reticular activation, blocks convulsive activity, while reticular deactivation leading to slow cortical waves, tends to facilitate epilepsy. as happens in the SW stage of sleep. Your "killer cats" were more attentive and with a more desynchronized cortex than the "non-killer" group. Dr. Adamec: Yes, I agree. In future studies. I intend to try to monitor perhaps telemetrically the responses in all of these areas while the animal is actively interacting with a prey. This approach may yield more consistent data in the secondary areas. One point I ought to mention. is that I was recording differentially and bipolarly. This may be a problem in some of the secondary areas. One case in point for which there are some data is the ventromedial hypothalamus. Tsubokawa and Sutin have evidence that there are cells localizing in the ventromedial hypothalamus which respond to basomedial amygdala stimulation and the distribution of these cells is fairly uniform. Since I am differentially recording I might not really record any potential difference within a homogeneously activated structure. $D r$. Tanaka: When certain areas such as the mesencephalic reticular formation are stimulated with high frequency an extremely arousing effect was elicited and the seizure was triggered, but when the central grey matter was stimulated with low frequency, it provoked tranquility and a quiet resting position even if the animals were a little irritable after attachments of the cable to the head. When dipropylacetate was administered to "killer Cats" they changed to "non-killer Cats" according to the work of our French group. We can also suggest a differential GABA stimulation of central grey matter by this information. My question is: If it is necessary to have such a prolonged stimulation or prolonged afterdischarge to obtain a kindled effect in "nonkiller" animals, don't you think that it might be worthwhile to know the possible role of GABA in "non-killer" or "killer" animals. Dr. Adamec: Yes, I do. Dr. Mclntyre: I never have understood whether you are getting interictal discharge in these animals or not. $D r$.
Adamec: I have to admit that I did not analyse it in great detail. All I can give you is an impression. There is some interictal discharge appearing afterwards but it disappears even though the behavior remains suppressed. From my experience at this conference. I will certainly do a detailed analysis in the future studies.

\section{ACKNOWLEDGEMENTS}

Gratitude is more than due to my wife Cannie for her help and perseverance during an often arduous endeavour and to Douglas Hamilton for his efforts in analyzing the neurosensory response data. The help of Michele Pontbriand in the food deprivation studies was also invaluable.

This work was supported by the Advanced Research Projects agency of the United States Department of Defence and monitored by the Office of Naval Research under contracts number N00014-70-C-0350 and the National Research Council grant number A7891, while a graduate student of Ronald Melzack at McGill University. Preparation of the manuscript was done while a research associate of G. V. Goddard at Dalhousie University with the support of the National Research Council grant number APA 365.

\section{REFERENCES}

ADAMEC, R. (1974a). Neural Correlates of Long Term Changes in Predatory Behavior in the Cat. Ph.D. Dissertation, McGill University.

ADAMEC, R. (1975). Naturally occurring physiological differences in the limbic systems of killer and non-killer cats. Agressive Behavior, Vol. 3.

ADAMEC, R. and HIMES, M. The effects of food deprivation on the mouse killing behavior of rats. Paper presented at the Canadian Psychological Association, June, 1975.

ALONSO DE FLORIDA, F. A. and DELGADO, J. M. R. (1958). Lasting behavioral and EEG changes in cats induced by prolonged stimulation of the amygdala. American Journal of Physiology, 193, 223.

DeFEUDIS, P. (1974). An analysis of the predatory behavior of the rat. Ph.D. Dissertation, McGill University.

DELGADO, J. M. R. and SEVILLANO, M. (1961). Evolution of repeated hippocampal seizures in the cat. EEG and Clinical Neurophysiology, 13, 722 .

DOUGLAS. R. M. and GODDARD, G. V. (1975). Long-term potentiation of the perforant path-granule cell synapse in the rat hippocampus. Brain Research, 86, 205.

FLYNN, J. P. (1967). The neural basis of aggression in cats. In D. C. Glass (Ed.), Neurophysiology and Emotion. New York: The Rockefeller University Press.

FLYNN, J. P., VANEGAS, H., FOOTE, W. and EDWARDS, S. (1970). The neural mechanisms involved in a cat's attack on a rat. In R. E. Whalen, R. F. Thompson, M. Verziano \& N. M. Weinberger (Eds.), The Neural Control of Behavior. New York: Academic Press.
GODDARD, G. V. and DOUGLAS, R. (1975). Does the engram of kindling model the engram of long-term memory.

GROSSMAN, S. P. (1963). Chemically induced epileptiform seizures in the cat. Science, 142, 409.

HAPPEL, L. T. and BACH, L. M. (1970). Amygdalopetal fiber influences upon excitability of amygdaloid nuclei. Federation Proceedings, 29, No. 829 (abstract).

HEBB, D. O. (1949). The Organization of Behavior. New York: Wiley.

HINDE, R. (1966). Animal Behaviour. Toronto: McGraw-Hill.

KAADA, B. (1972). Stimulation and regional ablation of the amygdaloid complex with reference to functional representations. In B. E. Eleftheriou (Ed.), The Neurobiology of the Amygdala. New York: Plenum Press.

KUO, Z. Y. (1930). The genesis of the cat's responses to the rat. Journal of Comparative Psychology, 11, 1.

LEYHAUSEN, P. (1956). Vehaltensstudien an Katzen. Z. Tierpsychol. Beiheft, 2, I.

MCINTYRE, D. C. and MOLINO, A. (1972). Amygdala lesions and CER learning: Long-term effect of kindling. Physiology and Behavior, 8, 1055.

O'KEEFE, J. and BOUMA, H. (1969). Complex sensory properties of certain amygdala units in the freely moving cat. Experimental Neurology, 23, 384.

PAUL, L., MILEY. W. M. and BAENNINGER, R. (1971). Mouse killing by rats: roles of hunger and thirst in its initiation and maintenance. Journal of Comparative and Physiological Psychology, 76(2), 242.

RACINE, R. J. (1972a). Modification of seizure activity by electrical stimulation: 1 . After-discharge threshold. EEG Clinical Neurophysiology, 32, 269.

RACINE, R. J., OKUYAUA, V. and CHIPASHVLI, S. (1972b). Modification of seizure activity by electrical stimulation: III. Mechanisms. EEG Clinical Neurophysiology, 32, 295.

RACINE, R. J.. GARTNER, J, G. and BURNHAM, W. M. (1972). Epileptiform activity and neural plasticity in limbic structures. Brain Research, 47, 262.

SIEGAL, A. and FLYNN, J. P. (1968). Differential effects of electrical stimulation and lesions of the hippocampus and adjacent regions upon attack behavior in the cat. Brain Research, 7, 252.

STOKMAN, C. L. J. and GLUSMAN, M. (1970). Amygdaloid modulation of hypothalamic flight in cats. Journal of Comparative and Physiological Psychology, 71, 365 .

VAN ATTA, L. and SUTIN, J. (1972). Relationships among amygdaloid and other limbic structures influencing activity of lateral hypothalamic neurons. In B. E. Eleftheriou (Ed.), The Neurobiology of the Amygdala. New York: Plenum Press.

WINER, B. (1962). Statistical Principles in Experimental Design. Toronto: McGrawHill. 